\title{
Backhauling in TV White Spaces
}

\author{
Cyrus Gerami, Narayan Mandayam, Larry Greenstein \\ WINLAB, Rutgers University \\ 671 Route 1 South, North Brunswick NJ 08902 \\ Email: \{cgerami, narayan, ljg\}@winlab.rutgers.edu
}

\begin{abstract}
Since the FCC's approval of unlicensed use of TV white spaces, the issue of how to use these white spaces has led to innovative technologies such as cognitive radios as well as a variety of spectrum policy proposals. There have been proposals to devise alternate rules for spectrum usage citing the overly conservative restrictions on secondary transmissions to protect incumbents. In this paper, instead, we propose to utilize white spaces for a backhaul network for internet traffic based on existing restrictions. Using the available white spaces and backhaul traffic demands in New Jersey as a case study, we evaluate the feasibility of such backhauling and present a methodology that can be used for other areas as well. Using a basic design involving fixed towers and directional antennas, our results show that the TV white spaces can be an effective medium for radio backhaul as an alternative to the costly laying of optical fiber. We also show that meeting FCC requirements on sensing and avoiding harm to wireless microphones will have only a minor impact on capacity.
\end{abstract}

\section{INTRODUCTION}

$\mathbf{T}$ He FCC's opening up of TV white spaces for unlicensed use has led to innovations in cognitive radio technology [1], spectrum sensing [2] as well as novel proposals for dynamic spectrum access [3]. In its Second Report and Order [4], the FCC set forth rules for TVBDs (TV Band Devices) by allowing transmission only in certain channels (see Table I). To further protect the licensed TV towers, FCC has also set forth rules for secondary antennas and transmission power, which in turn restricts the transmission coverage for a "secondary-tosecondary" system. These restrictive requirements have raised several objections and have also resulted in proposals for alternative rules that can still provide sufficient protection to the incumbents (e.g. [5]). To date, in spite of these objections, nothing has changed; the rules remain the same.

In this paper, we address the following question: How can one use the TV white spaces within the existing rules? While proposals have been set forth for use of white spaces for local area networks [6], one potential use which might be of interest is a backhaul network for rural areas and areas with no pre-existing wired infrastructure. We propose a system using fixed towers and directional antennas which would provide backhaul and/or distribution for internet access in places where it is needed and is presently not available. Using New Jersey as a "testbed", we calculate the available white space and backhaul traffic demands and evaluate the feasibility of such backhauling and present a general methodology that can be used for other areas as well.

While optical transport networks are evolving rapidly [7], [8], having ubiquitous deployment of these, including in rural
TABLE I: Allowable Channels

\begin{tabular}{cccc}
\hline \hline TV Channels & Frequency Band & Frequency $(\mathrm{MHz})$ & Allowed Devices \\
\hline 2 & VHF & $54-60$ & Fixed \\
$5-6$ & VHF & $76-88$ & Fixed \\
$7-13$ & VHF & $174-216$ & Fixed \\
\hline $14-20$ & UHF & $470-512$ & Fixed \\
$21-35$ & UHF & $512-602$ & Fixed and Portable \\
36 & UHF & $602-608$ & Portable \\
38 & UHF & $614-620$ & Portable \\
$39-51$ & UHF & $620-698$ & Fixed and Portable \\
\hline
\end{tabular}

areas, is far from being a reality. The backhaul network concept we propose is an attractive alternative to laying optical fiber, which is costly, while radio towers using TV white spaces is far less so. New Jersey is an appropriate testbed for evaluating this concept. It is the most densely populated state in the US; has large pockets of urban, suburban and rural areas; and is adjacent to two large metropolitan areas-New York and Philadelphia.

The rest of this paper is organized as follows: In Section II, our methodology is outlined and the available TV white space spectrum in the UHF channels for fixed TVBDs is mapped to the geography of New Jersey. In Section III, we discuss issues related to wireless microphones and avoiding interference to them. In Section IV, we describe our method for calculating achievable capacity in the links of our proposed network. In Section V, we compare the achievable rate and the demand in each part of the state. Our findings indicate that the proposed backhaul network in the TV white spaces is feasible, and any further relaxation of the FCC rules for white space transmission can only enhance the performance of such wireless backhauling.

\section{Methodology}

To quantify white space availability in New Jersey, we first divide the state into a grid of $5 \mathrm{mi}$ x $5 \mathrm{mi}$ square cells, as shown in Fig. 1a. Now assuming there is a radio tower in (or near) the middle of each of these cells, the distance to transmit data from one cell to another would be 5 miles. We also assume that each radio tower has 4 sector antennas covering all directions instead of one isotropic antenna, as shown in Fig. 1b. This allows more concentrated line-of-sight (LOS) transmission and less interference.

To determine whether TV white spaces can be used for backhaul, we need to compare the achievable capacity for each link with the demand for service in each square cell. Achievable capacity will be derived using FCC power limits 


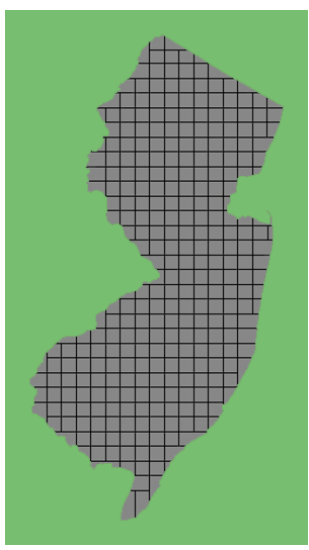

(a) A proposed grid of 307 square cells, each 5 miles x 5 miles.

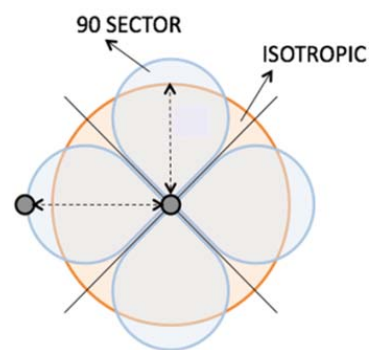

(b) A plan for using foursector antennas at each radio tower.
Fig. 1: Using radio radio towers for internet backhaul in New Jersey

and widely accepted propagation models. Traffic demand per cell will be derived using a Cisco data traffic survey [9].

If the deliverable capacity is less than the demand in any area, connectivity cannot be provided by utilizing the white spaces and fiber would be needed instead. Interestingly, the areas in which fiber would be needed are found here to be those already provided with wired service. We also find, as we will show, that in the vast majority of cells in New Jersey, the deliverable capacity is greater than the likely demand.

A major step towards finding the achievable rates is to map the allowed channels to New Jersey's geography, in order to calculate the available bandwidth per cell-to-cell link. We do that next.

Other than a list of allowed channels for secondary transmission, the FCC has set a protected radius $\left(r_{p}\right)$ around each licensed TV tower in which no secondary transmission is allowed. On top of that, for fixed devices (base-stations or towers), co-channel or even adjacent-channel transmission is not allowed. Also, to compensate for the interference introduced by the secondary radio towers, FCC has set an extension to the protected radius for co-channel transmission as well as adjacent channel transmission. Figure 2 shows the protected radius, the additional separation radius and the noise limited radius around a TV tower in comparison with a secondary

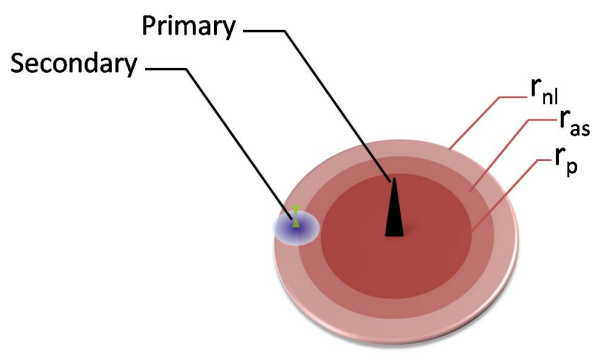

Fig. 2: A primary radio tower with its protected zone, and a secondary radio tower with its allowed coverage. See text for more details. antenna and its presumed coverage.

In order to build up a database of available channels per square cell in New Jersey, we used Google maps and implemented all FCC restrictions on all TV towers in New Jersey along with all TV towers in New York, Pennsylvania and Delaware which had coverage extending into New Jersey [10]. We include these three states because a given TV tower's coverage can span up to $100 \mathrm{~km}$, which can limit use of the same channel in a neighboring state.

After mapping these rules for all allowed UHF channels (i.e. channels available from channel 14 to 51, as shown in Table I) to our grid, we have the available channels per square cell. Figure 3 illustrates a geographical map of available channels for fixed TVBDs, where the degree of shading indicates the number of channels available. In this case, the minimum number of available channels is 7 and the maximum is 31 . This would mean that at every point in New Jersey as least $42 \mathrm{MHz}$ of bandwidth (7 channels of 6-MHz bandwidth) is available for unlicensed use. Also note that for this exercise, only full-power TV stations have been considered and inclusion of low-power TV, PLRMS/CMRS and other licensed services would slightly reduce the channel availability in some localities. Nevertheless, the number of available channels from our calculations is comparable to those obtained using online databases such as [11] and [12].

\section{WIRELESS MICROPHONES}

Any scheme for using white spaces, including the one proposed here, must be able to sense wireless microphones (WMs) and shut down transmission in TV channels where they are detected. The operative definition of 'detected' is that the received power is measured to be at or above -114 $\mathrm{dBm}$ when using an isotropic antenna mounted $10 \mathrm{~m}$ or higher above ground. For our system in New Jersey, we assume an antenna height $30 \mathrm{~m}$ above ground and make a first-order (conservative) calculation of the number of detectable WMs per radio tower.

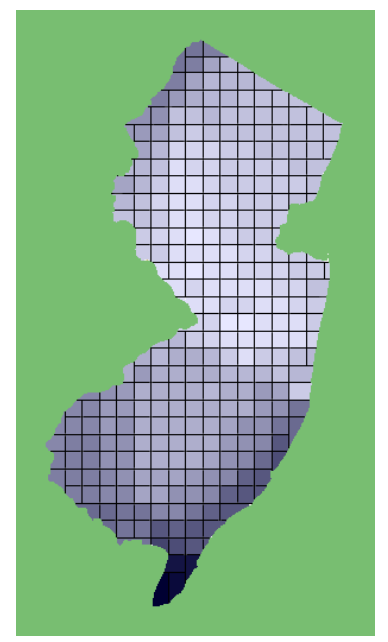

Fig. 3: Number of available 6-MHz channels within the 5-mile $\mathrm{x}$ 5-mile square cells, where the darkness of the shadings represents the number of channels. For New Jersey, the number ranges from 7 (lightest shading) to 31 (darkest shading). 
A typical WM transmits at a power of $50 \mathrm{~mW}(17 \mathrm{dBm})$, so a path loss above $131 \mathrm{~dB}$ would yield received WM signals below threshold. To estimate path loss vs. distance, we use Hata's Suburban model [13] in the UHF band, and we add 10 $\mathrm{dB}$ for transmission loss into buildings, a very conservative increment according to most reports (cf. [14]). The result is that ground-level WMs inside buildings would be detectable within a 1-mile radius around our radio towers. The radius would be even smaller using Hata's Urban model and/or a higher (and more likely) transmission loss into buildings.

Next, we estimate the expected number of WMs in a 1-mile radius, i.e., within an area of 3.14 square miles, in New Jersey. According to [15] and [16], there are about 2500 schools and 4500 churches in the state, and these are assumed here to be the dominant WM users. Given a total area of 7836 square miles, this translates to averages of 1 school and 1.8 churches per 1-mile radius. Thus, at most three $6-\mathrm{MHz}$ channels might require shutdown in a typical cell at a given time, which is small compared to the number of channels available per cell (7-31, as noted above).

Several additional ameliorating factors can be noted. First, the affected WMs in a given cell may congregate in one or two channels rather than three; second, data on school and church locations could be used to advantageously tailor the positions of radio towers; third, the focus of our study is rural areas, where the densities of schools and churches may be below the average; fourth, peak-hour usages of WMs in schools and churches (daytimes and evenings, respectively) differs from those for internet users ( $9 \mathrm{pm}-1$ am [9]), thus reducing the 'duty cycle' of channel shutdowns needed to protect WMs. We conclude that the unavailability of channels due to WMs would have a minor impact on the performance of our proposed system.

Aside from meeting FCC requirements, we believe that our system would pose little potential danger to WM usage. This is because of the height, directionality and fixed positions of the radio tower antennas, and the fact that most WMs would be near the ground. A more detailed study would be needed to confirm this result or to engineer around potential problems. At this stage, however, ignoring channel shutdowns due to WMs seems to be a reasonable working assumption.

\section{Achievable Capacity Calculations}

In order to compute the achievable capacity, we assume that all TV white space transmitters (towers) have the ability to execute perfect spectrum sensing and avoid causing any interference to any of the primary (protected) devices using this spectrum. We calculate the achievable capacity based on the available bandwidth and spectrum efficiency achieved by these TV white space transmissions. The available bandwidth was described in Section II. For determining the spectral efficiency, we need the received signal-to-noise ratio (SNR) of the TV white space transmission. The SNR at the receiving antenna depends on the transmission power, path loss and noise. The transmission power for fixed devices has been limited by the FCC to 4 watts $(6 \mathrm{dBW})$ and noise consists of thermal noise augmented by the receiver noise figure, assumed here to be $10 \mathrm{~dB}$.
Path loss: To determine the path loss in our 5-mile LOS links, we use the ITU Terrain Propagation Model [17]. This model is ideal for LOS transmissions with regular obstructions on the path. Under this model the median path loss $\left(P L_{50 \%}\right)$ is given by

$$
P L_{50 \%}=F S L+A_{d} \quad d B,
$$

where FSL is the free-space path loss given as

$$
F S L=20 \log _{10} d+20 \log _{10} 1000 f+32.44 \quad d B .
$$

The inter-terminal distance $d$ is in $\mathrm{km}$, the frequency $f$ is in $\mathrm{GHz}$, and $A_{d}$ is the excess path loss above and beyond freespace loss. It is based on the antenna height, the obstruction height and the link distance, and is given by

$$
A_{d}=-20 h / F_{1}+10 \quad d B,
$$

where $h$ is the height difference between the most significant path blockage and the line-of-sight path between the transmitter and the receiver; and $F_{1}$ is the radius of the first Fresnel zone given by

$$
F_{1}=17.3 \sqrt{\frac{d}{4 f}} \quad m,
$$

where $d$ is in $\mathrm{km}$ and $\mathrm{f}$ is in GHz. Note that the obstruction is considered to be at the mid-point between the two antennas.

To be on the conservative side, we add a dB term to the path loss to account for random variations about the ITU prediction; and we model this term as a Gaussian variate of zero mean and standard deviation $\sigma$. Also, to be conservative, we assume the random variation term is at its $99 \%$ value on all links (only $1 \%$ of all links have a greater value), in which case we can write the path loss on every link as

$$
P L_{\text {total }}=P L_{50 \%}+2.3 \times \sigma \quad d B .
$$

SNR and Spectral efficiency: The received path loss can be written as

$$
S N R=P_{T}-10 \times \log _{10}(k T B)-N_{F}-P L_{\text {total }} \quad d B,(6)
$$

where $P_{T}$ is transmit power, $B$ is bandwidth ( $\left.6 \mathrm{MHz}\right), k T$ is thermal noise density, and $N_{F}$ is the receiver noise figure (10 $\mathrm{dB})$.

The theoretical upperbound, $\eta$, on spectral efficiency is given by

$$
\eta=\log _{2}\left(1+10^{\frac{S N R}{10}}\right) \quad \text { bps } / H z
$$

The parameters used for our analysis are given in Table II. Note that we consider two different values for obstruction height; this is due to different structure heights in cities and is based on population. In our model, we have assumed $15 \mathrm{~m}$ obstruction heights (5-story buildings) for sparsely populated areas, and $30 \mathrm{~m}$ heights (10-story buildings) for densely populated areas. Also, note that some parameters in Table II 
TABLE II: Parameters used in calculations

\begin{tabular}{r|l}
\hline \hline Link height & 30 meters \\
\hline Obstruction height & $\begin{array}{l}30 \text { meters (for densely populated areas) } \\
15 \text { meters (for sparsely populated areas) }\end{array}$ \\
\hline Link distance $(\mathrm{d})$ & 5 miles (8 kilometers) \\
\hline Frequency $(\mathrm{f})$ & $580 \mathrm{MHz}$ (average white space frequency) \\
\hline Transmission power $\left(P_{T}\right)$ & $6 \mathrm{dBW}(4$ watts) \\
\hline Thermal noise $(\mathrm{kTB})$ & $-136 \mathrm{dBW}$ \\
\hline Noise Figure $\left(N_{F}\right)$ & $10 \mathrm{~dB}$ \\
\hline Shadowing margin $(\sigma)$ & $3 \mathrm{~dB}$ \\
\hline
\end{tabular}

are specified by the FCC and others are typical numbers used in similar studies.

The effect of obstruction height on path loss in the ITU model is illustrated in Fig. 4, which plots SNR vs. obstruction height for different frequencies. Now, for the two different obstruction heights, we can compute the SNRs and the corresponding spectral efficiencies as follows:

$$
\begin{aligned}
& h_{o}=15 \mathrm{~m} \Rightarrow S N R=18.7 \mathrm{~dB} \Rightarrow \eta=6.23 \mathrm{bps} / \mathrm{Hz} \\
& h_{o}=30 \mathrm{~m} \Rightarrow S N R=9.3 \mathrm{~dB} \Rightarrow \eta=3.25 \mathrm{bps} / \mathrm{Hz}
\end{aligned}
$$

To determine where to assume a sparse population and where to assume a dense one we estimated the population for each cell based on US Census 2000 data for population density in New Jersey [18]. The shadings of Fig. 5 indicate the population distribution of New Jersey based on our grid. Frequency planning: Most available channels are similar in neighboring cells. Thus, when using same channels in neighboring cells, we will have co-channel interference problems. In an array of cells, frequency planning would insure that the receiving terminal in a link receives a relatively higher signal power from its corresponding transmitter rather than any other neighboring transmitter. In other words, in Fig. 6, if cell 1 is

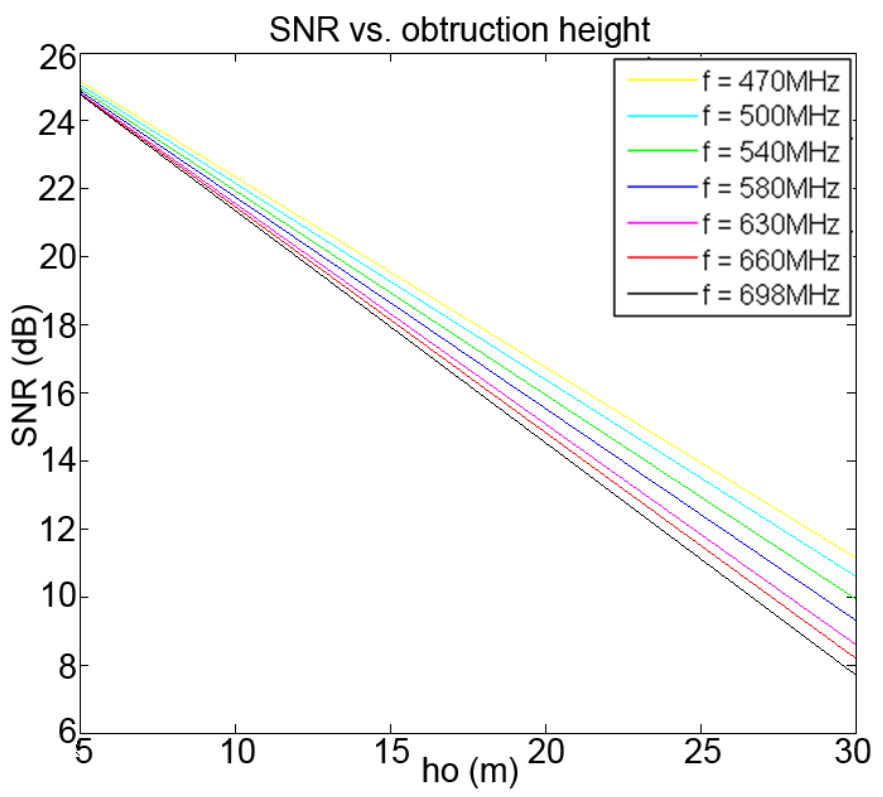

Fig. 4: Received SNR vs. obstruction height on a 5-mile path, for different frequencies. This plot is based on the ITU path loss model, with the obstruction assumed to be at the center of the path.

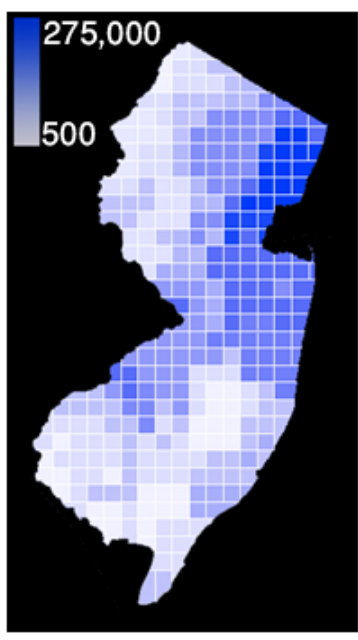

Fig. 5: Population distribution in New Jersey, based on the US Census of 2000. Darker shadings correspond to higher densities, with the darkest areas concentrated in the New York City metropolitan area.

transmitting to cell 2 in a certain channel, cell 2 cannot use that channel to transmit to cell 3 . To guarantee this, cells 1 and 2 should transmit on different channels. For example, with a frequency reuse factor of 2, cells 1 and 3 would both use frequency $f_{a}$ but not $f_{b}$, and cells 2 and 4 would both use frequency $f_{b}$ but not $f_{a}$. Note that cell 4 will receive signals from cells 1 and 3, though the latter signal will be stronger by about $14.5 \mathrm{~dB}$ (see lower part of Fig. 6, which shows the decay of the received SNR with distance, using our path loss model).

To alleviate co-channel interference (e.g., the interference to the cell 4 receiver from the cell 1 transmitter), we can assume that each tower has a second receive antenna, used to effect interference cancellation. Alternatively, we can assume a larger reuse factor ( 3 or higher), at a cost in capacity. In our calculations here, we will assume that a second receive antenna

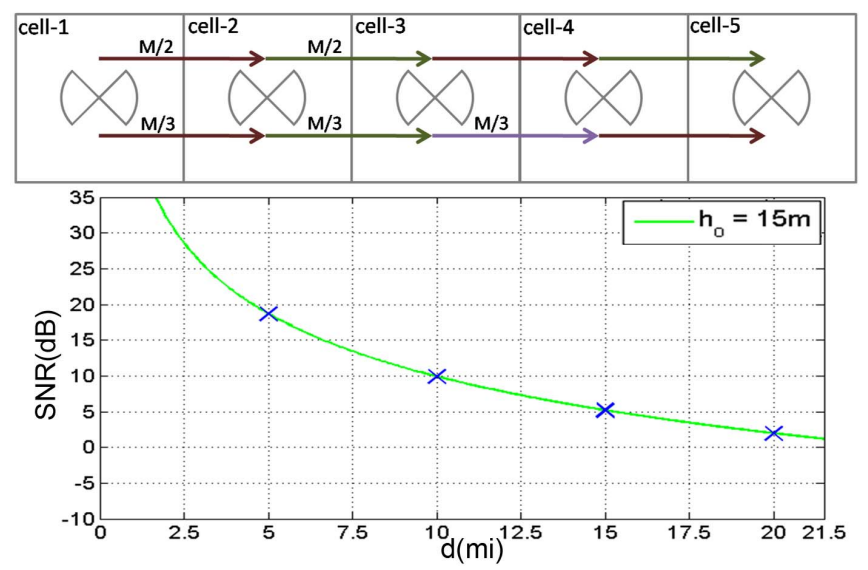

Fig. 6: (top) five neighboring cells with $\mathrm{M}$ common available channels illustrating usage of different sets of channels with different colors for a reuse factor of 2. (bottom) a plot of the received SNR from cell 1 vs. distance, illustrating the fading of the signal power as it reaches cell 3. Each ' $\mathrm{X}$ ' on the plot represents the position of neighboring receivers. 
is used to cancel interference, and that the reuse factor is 2 . Under these assumptions, we can construct a database for the maximum deliverable rate from each cell.

\section{DEMAND}

To characterize the internet traffic demand in each location in New Jersey, we use a Cisco survey on internet traffic [9]. This survey says that an average broadband connection generates 11.4 GB traffic per month (375 MB per day per connection). In peak hours generated traffic is $18 \mathrm{MB}$ per connection per hour in comparison to nonpeak hours in which generated traffic in $15 \mathrm{MB}$ per connection per hour. In addition, Cisco has estimated that peak internet traffic may grow seven-fold by 2013, compared to a five-fold increase of average Internet traffic.

In our model, we used this information to estimate a futuristic demand. We take into consideration two parameters: usage per household and simultaneous active users.

Usage per household: We have the Year 2000 population data for each cell of the grid shown for New Jersey. The average population per household in the US is 2.48 [19], which we round up to 3. Also, $74.2 \%$ of people in the US have internet access [20]. From these estimates, we can estimate the number of internet clients in each cell. In making this calculation, we assume a current peak traffic of $18 \mathrm{MB} /$ hour, with a potential future growth in traffic by factors of 5, 7 and 10 (corresponding to 90,126 and $180 \mathrm{MB} /$ hour in the future).

Active users: We parameterize the percentage of active households for any given time and take 10\%, 30\% and 50\% active households as three different scenarios.

Based on these two parameters, we can construct a database, consisting of the demand for different hourly traffics and different active user percentages for each cell.

\section{Vi. Feasibility of a Backhaul Network in New JERSEY}

A comparison of achievable rates and demand per cell lead us to some findings on the feasibility of our proposed backhaul system. If the achievable capacity is less than demand in a given cell, fiber would be needed. Otherwise, radio towers and TV white spaces could be used to transfer traffic between neighboring cells.

Table III shows the number of cells (out of a total of 307 cells) that would need fiber connectivity to its neighbors, under our study assumptions. Also, Fig. 7 illustrates the geographical distribution of these cells on a New Jersey map, assuming $30 \%$ simultaneous active users.

Note that most places that cannot be supported by using radio towers and white spaces are in the New York or Philadelphia metropolitan areas. It is safe to assume, therefore,

TABLE III: Number of cells that need fiber out of a total 307 cells

\begin{tabular}{|c|c|c|c|}
\hline \hline per hour usage & $10 \%$ active & $30 \%$ active & $50 \%$ active \\
\hline $18 \mathrm{MB}$ & 20 & 63 & 70 \\
\hline $90 \mathrm{MB}$ & 70 & 117 & 137 \\
\hline $126 \mathrm{MB}$ & 80 & 135 & 141 \\
\hline $180 \mathrm{MB}$ & 91 & 139 & 155 \\
\hline
\end{tabular}
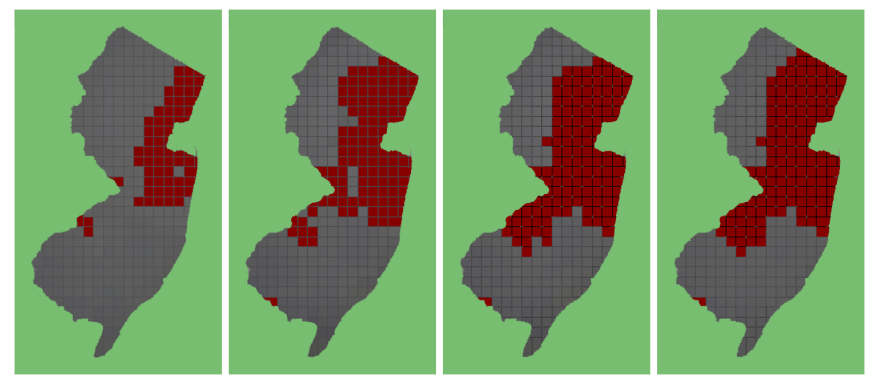

Fig. 7: Geographical distribution of cells needing fiber, assuming $30 \%$ active users. The usage per house, from left to right, is 18,90 , 126 and $180 \mathrm{MB}$ per hour

that these areas already have a fiber infrastructure in place. Conversely, all of the areas which are somewhat remotely located, basically places that may not have access to wired infrastructure, do have ample TV white space capacity making wireless backhauling a viable proposition.

\section{CONCLUSION}

In this paper, we have proposed to utilize white spaces for a backhaul network for internet traffic based on existing FCC restrictions. Using the available white spaces in the UHF channels for fixed TVBDs and backhaul traffic demands in New Jersey for our case study, we have evaluated the feasibility of such backhauling and have presented a methodology that can be used for other areas as well. Our results show that the TV white spaces can be an effective medium for radio backhaul as an alternative to the costly laying of optical fiber.

Most parameters used in our calculations are conservative, with results that are correspondingly pessimistic. Under these conservative assumptions, and for the most extreme conditions considered, only $50 \%$ of New Jersey cannot be covered using radio towers and TV white spaces. This is a very satisfying result, leading to a positive finding for the proposed backhaul concept. Further, any future relaxation of the FCC rules for white space transmission will only enhance the performance of such wireless backhauling. In addition, the methodology used here can be transported to other cases (e.g., other states) to see if the same concept can be beneficially applied elsewhere.

The next round of investigation should go deeper into the issue of wireless microphones, and also examine the cost issues in detail to estimate how much the proposed system could save. Another issue, not addressed in this initial study, is the potential for conflict with other secondary users of the white spaces.

\section{ACKNOWLEDGMENTS}

The authors thank Dipankar Raychaudhuri, Ivan Seskar and Richard Frenkiel for the early conversations on the idea of a radio backhaul network using TV white spaces and also, Paul Henry and Chris Rose for their useful comments. 


\section{REFERENCES}

[1] I. Akyildiz et al., "A survey on spectrum management in cognitive radio networks," IEEE Commun. Mag., vol. 46, no. 4, pp. 40 -48, Apr 2008.

[2] S. J. Shellhammer, "Spectrum sensing in ieee 802.22," IAPR Wksp. Cognitive Info. Processing, Jun 2008.

[3] O. lleri and N. B. Mandayam, "Dynamic spectrum access models: toward an engineering perspective in the spectrum debate," Communications Magazine, IEEE, vol. 46, no. 1, pp. 153 -160, Jan 2008.

[4] "In the matter of unlicensed operation in the tv broadcast bands: Second report and order and memorandum opinion and order," Nov 2008. [Online]. Available: http://hraunfoss.fcc.gov/edocs public/attachmatch/FCC-08-260A1.pdf

[5] S. M. Mishra and A. Sahai, "How much white space is there?" Tech. Rep. EECS-2009-3, Jan 2009. [Online]. Available: http://www.eecs.berkeley.edu/Pubs/TechRpts/2009/EECS-2009-3.html

[6] P. Bahl et al., "White space networking with wi-fi like connectivity," in Proceedings of the ACM SIGCOMM conference on Data communication, New York, NY, USA, Aug 2009, pp. 27-38.

[7] J. D'Ambrosia, "100 gigabit ethernet and beyond [commentary]," Communications Magazine, IEEE, vol. 48, no. 3, pp. S6 -S13, Mar 2010.

[8] G. Wellbrock and T. Xia, "The road to $100 \mathrm{~g}$ deployment [commentary]," Communications Magazine, IEEE, vol. 48, no. 3, pp. S14 -S18, Mar 2010.

[9] "Cisco visual networking index study on peak internet traffic," 2009. [Online]. Available: www.cisco.com/web/go/vni

[10] "List of all class a, lptv, and tv translator stations," 2008. [Online]. Available: http://www.dtv.gov/MasterLowPowerList.xls

[11] "List of licensed tv channels available at each location." [Online]. Available: www.tvfool.com

[12] "List of available channels for unlicensed use," 2010. [Online]. Available: www.showmywhitespace.com

[13] M. Hata, "Empirical formula for propagation loss in land mobile radio services," IEEE Transactions on Vehicular Technology, vol. 29, no. 3, pp. $317-325$, aug 1980 .

[14] T. S. Rappaport, "Wireless communications: Principles and practices," IEEE Press and Prentice Hall, p. 124, 1996.

[15] "New jersey public schools fact sheet." [Online]. Available: http://www.state.nj.us/education/data/fact.htm

[16] "New jersey christian church directory." [Online]. Available: http://www.churchangel.com/newjersy.htm

[17] "Itu-r recommendations." [Online]. Available: http://www.itu.int/publ/RREC/en or http://en.wikipedia.org/wiki/ITU Terrain Model

[18] "Us census 2000 gazetteer files." [Online]. Available: http://www.census.gov/geo/www/gazetteer/places $2 \mathrm{k} . h t m l$

[19] "State and county quickfacts." [Online]. Available: http://quickfacts.census.gov/qfd/meta/long HSD310200.htm

[20] "Usage and population statistics." [Online]. Available: http://www.internetworldstats.com/ 